\title{
A Framework to Relieve Wireless Hot-Spot Congestion by Means of Ad Hoc Connections
}

\author{
Jiancong Chen ${ }^{\dagger} \quad$ Jingyi $\mathrm{He}^{\ddagger} \quad$ S.-H. Gary Chan ${ }^{\dagger}$ \\ ${ }^{\dagger}$ Department of Computer Science \\ ${ }^{\ddagger}$ Department of Electrical and Electronic Engineering \\ Hong Kong University of Science and Technology \\ Clear Water Bay, Kowloon, Hong Kong \\ Email: kentchen@cs.ust.hk eehjy@ust.hk gchan@cs.ust.hk
}

\begin{abstract}
In recent years, wireless public networks have been widely deployed to provide high-speed Internet access to mobile users. Due to the dynamic and uneven distribution of user requests, some access points (the so-called "hot-spots") may be congested by many users. In order to alleviate such congestion, we introduce an ad hoc relaying wireless LAN framework in this paper. A user can be served by a neighboring access point through an ad hoc connection in such a network. We propose a framework to establish such ad hoc connections. Using simulations, we show that the framework is efficient to reduce the service failure rate as well as to improve channel utilization in hot-spot networks.
\end{abstract}

\section{INTRODUCTION}

Wireless LANs (WLANs) are being widely deployed in offices, homes, as well as public areas like airports and malls, to provide high-speed Internet access to mobile users. A WLAN is set up by attaching to the wired network a base station (BS) (also called an access point, or AP), which relays the traffic between the wireless users and the wired network [1]. In public areas covered by a number of APs, the user load is in general dynamic and unevenly distributed among the APs. It is possible that some APs (so-called "hot-spots") are congested by many users and cannot admit more requests, while their neighboring ones are serving few users and the bandwidth is underutilized. In this paper, we propose and study serving a mobile user blocked in a hot-spot by a neighboring AP through ad hoc connections, thus alleviating the congestion at the hot-spot as well as improving the bandwidth utilization of the whole network.

We illustrate in Fig. 1 the basic idea of the proposed scheme. In the example, three APs are considered, in which AP 1 is a hot-spot. The mobile host (MH) 1 in the coverage of AP 1 is blocked because there is insufficient bandwidth to satisfy its request requirement. As AP 3 is lightly-loaded and has enough free bandwidth, $\mathrm{MH} 1$ is directed to set up an ad hoc connection to $\mathrm{MH} 3$ which is in the coverage of AP 3. MH 3 maintains the ad hoc connection to MH 1 as well as its connection to AP 3 . In addition to communicating with AP 3 for its own traffic, MH 3 also operates as a relay agent for the communication between MH 1 and AP 3. In this way, MH 1 is served by (i.e., associated to) AP 3 in a two-hop manner, instead of being blocked. The major issue of the scheme is to find the appropriate relay agent to establish such an ad hoc connection for the blocked $\mathrm{MH}$.

This work was supported, in part, by the Competitive Earmarked Research Grant of Research Grant Council in Hong Kong (HKUST6014/01E and HKUST6199/02E).

†Correspondence author: Jiancong Chen

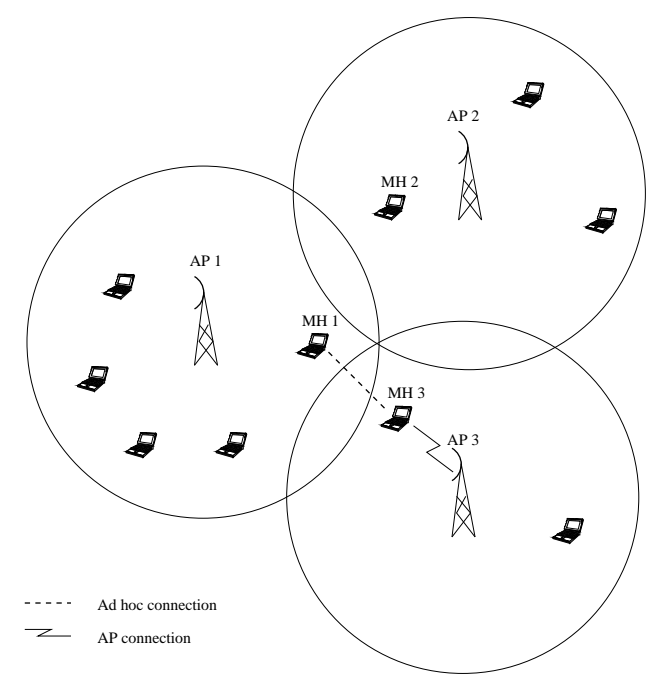

Fig. 1. An example of relieving hot-spot congestion through ad hoc connection.

In this paper, we propose and study a scheme called "APAssisted Passive Agent Selection" (APAS) for agent discovery and ad hoc connection establishment. With APAS, the neighboring APs cooperate to find out some possible agents for the blocked MH while the MH just waits for the connections from these agents and selects a proper one. The proposed scheme is based on the assumption that a mobile host ( $\mathrm{MH})$ can switch between infrastructure mode (i.e., communicating with the AP) and ad hoc mode (i.e., direct communication with other MHs), which has been shown able to significantly improve the performance of the WLAN [2].

We implement the framework of ad hoc relaying network in NS2 and use simulations to evaluate the performance. The performance metrics of interest are the service failure rate defined as the percentage of requests that cannot get their services and the bandwidth utilization in each AP. Our results show that with ad hoc relaying connections between neighboring APs, the service failure rate is reduced significantly and the system utilization is increased accordingly.

We briefly discuss previous work as follows. The congestion problem at WLAN hot spots has been studied in [3]. The authors proposed two approaches to relieve the congestion, namely, explicit channel switching and network-directed roaming. However, both of them have their limitations. Explicit channel switching only works for MHs in the overlap area of 
multiple APs, while network-directed roaming requires MHs to move around to get the access, which is neither convenient for the users nor reliable as network state might have changed after the roaming. Our proposed scheme of using ad hoc connections between MHs to balance the load between APs does not suffer from these limitations. A similar idea of using ad hoc connections to do load balancing between cells has been proposed for cellular networks [4], [5]. As the cellular system uses different frequency band than the mobile ad hoc network, the performance improvement by introducing ad hoc connections to deal with congestion is obvious. Our study differs primarily in that we are considering a unified wireless system in one frequency band, in which the bandwidth is shared by all the communications, and the performance improvement by introducing ad hoc connections is not as obvious and deserves a separate study.

The rest of this paper is organized as follows. We present in Sect. II a scheme for agent discovery and ad hoc connection establishment. Simulation results illustrating the performance of the scheme is presented in Sect. III. We conclude in Sect. IV.

\section{AD HOC RELAYING WIRELESS LANS}

In the proposed ad hoc relaying wireless LANs, when a mobile host sends a service request to the access point, the AP has admission control to check whether it can provide the service or not. If not, instead of blocking the request, the AP tries to find a neighboring AP that can provide the service and divert the MH to it by an ad hoc connection. Several issues need to be addressed in this process, which we discuss in the following.

First, the original AP (hot-spot) should know whether there is a neighboring AP with sufficient resources for the request that it cannot admit. This is achieved by maintaining and exchanging state information among APs. Since the number of neighboring APs is small and all APs are connected to the wired network, the communication overhead is limited and does not affect the performance of the wireless network.

Second, if there is such an AP that can provide the service, the MH should be able to connect to it. Since the cover ranges of the APs are limited and may not be overlapping (depending on the deployment and layout of the public wireless networks), the MH may not be in the range of the neighboring AP, i.e., a direct (one-hop) connection is not possible. Therefore, an agent is needed to relay the traffic in between. Hence finding such an agent to establish an ad hoc connection is the major issue in ad hoc relaying WLANs.

Moreover, after the connection is established between the $\mathrm{MH}$ and the neighboring AP through an agent, since the connection between the $\mathrm{MH}$ and the agent works in ad hoc mode while the agent connects to its AP in infrastructure mode, a good packet forwarding method is important in order to achieve high performance. As discussed in our previous paper, the difference between infrastructure mode and ad hoc mode in the IEEE 802.11 standard is several parameters in the frames, and the behavior of the transmissions [2]. The agent is hence able to send and receive packets in both modes. Upon receiving a packet from the AP to the $\mathrm{MH}$, it forwards the packet in ad hoc mode. Likewise, the packets from the $\mathrm{MH}$ are forwarded in infrastructure mode to the AP.

\section{A. AP-Assisted Passive Agent Selection (APAS)}

In this paper, we mainly focus on the agent discovery and connection establishment schemes, illustrated in Figs. 2 and 3 , including the operation procedures at both the APs and the

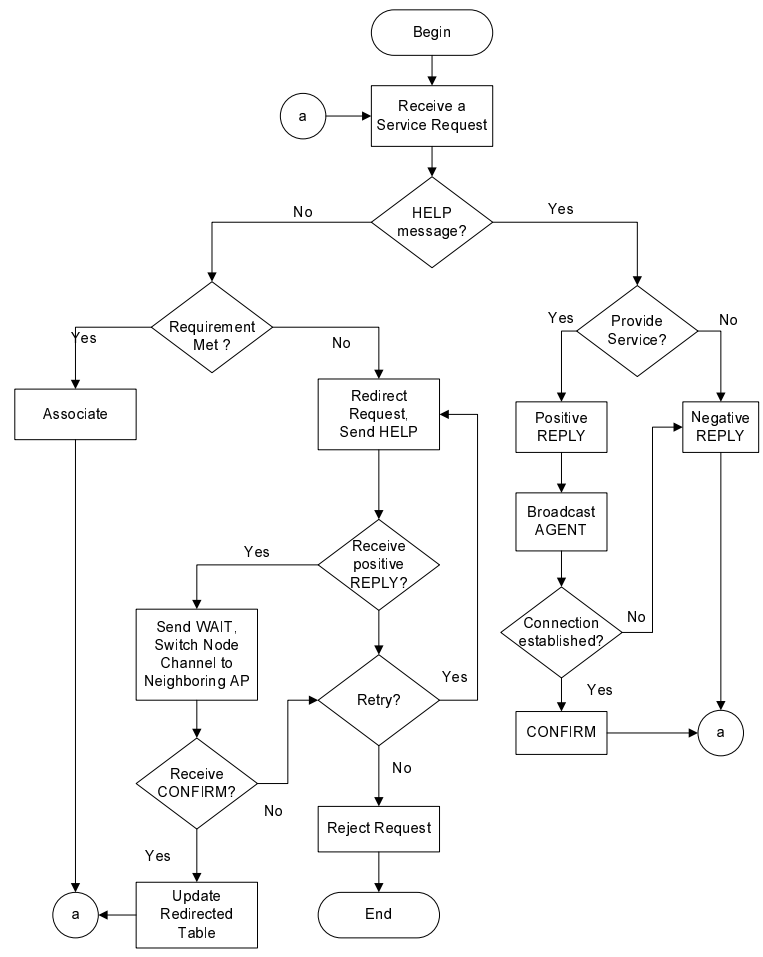

Fig. 2. The flowchart of ad hoc connection establishment algorithm at APs.

MHs. With APAS, the hot-spot AP forwards the (bandwidth) request of the blocked $\mathrm{MH}$ to its neighboring APs, which check if they have enough bandwidth to satisfy the request through a two-hop connection. Those having enough bandwidth indicate the MHs in their coverage to advertise the availability. The blocked $\mathrm{MH}$ listens to the advertisements for a while and selects the best one (which offers the largest bandwidth or is nearest in space) from the advertising agents within the communication range as the relay agent, and set up the ad hoc connection.

As mentioned before, when an AP receives a service request from a $\mathrm{MH}$, it first checks whether it has sufficient resources (termed residual bandwidth in this paper) to meet the requirement. If it is able to provide to service to the user, the user is then associated to it. If so, it sends "HELP" messages to its neighboring APs, including the information of the service requirement. The APs cooperate to find out which APs adjacent to the hot-spot AP can provide the service instead.

Upon receiving a "HELP" message, an neighboring AP responds with a positive "REPLY" message if it has enough resource to serve the request by a two-hop connection, or a negative one if otherwise. Then it broadcasts an "AGENT" message to the MHs associated to it. After a MH acting as an agent establishes an ad hoc connection to the redirected user, the AP sends a "CONFIRM" message back to the hot-spot AP.

After the hot-spot AP receives a "REPLY," it sends to the MH a "WAIT" message containing the channel information of the neighboring AP. The MH then switches to the channel and waits for the connection messages from the agents. Once the ad hoc connection is established, the hot-spot AP will receive the "CONFIRM" message from the neighboring AP. Otherwise, it repeats the above procedures to find another AP for the MH.

At a mobile host, if it receives a "WAIT" message after it sends a service request to an AP, it switches to the channel given by the message and listens in ad hoc mode for some time. After it receives some "AGENT" messages, it selects the agent with 


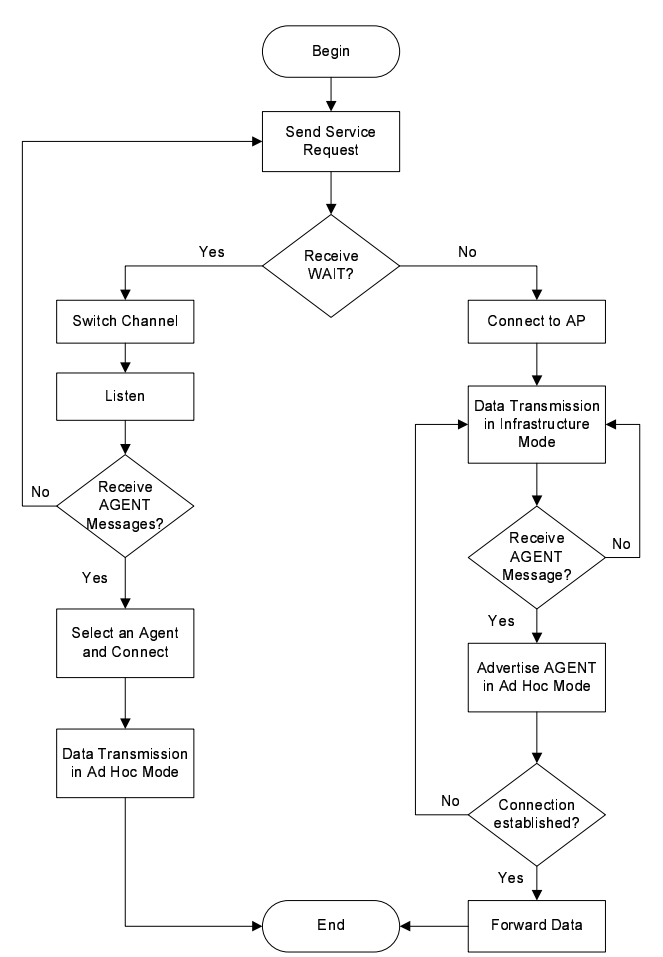

Fig. 3. The flowchart of ad hoc connection establishment algorithm at hosts.

the strongest signal power to connect to the neighboring AP. On the other hand, when an agent receives an "AGENT" message from its AP, it advertises the message in ad hoc mode so that the redirected $\mathrm{MH}$ can receive it.

As an example, refer to Fig. 1 again, the detailed steps of the connection establishment procedures are listed as follows.

1) A user ( $\mathrm{MH} \mathrm{1)}$ sends a service request to its AP (AP 1);

2) AP 1 finds out that the requirement can not be met;

3) AP 1 sends "HELP" messages containing the request information to its neighboring APs;

4) Once an AP (AP 3) receive a "HELP" message, it checks whether it can provide service to the request by a two-hop connection and responds with a "REPLY";

5) After AP 1 receives a positive "REPLY" message, it sends a "WAIT" message to MH 1;

6) MH 1 switches to the channel given by the "WAIT" message and listens;

7) After sending a positive "REPLY" to AP 1, AP 3 sends a "AGENT" message to the agents within the coverage;

8) MH 3 advertises the "AGENT" message;

9) Upon receiving the "AGENT" message, MH 1 selects an agent (MH 3). The connection is hence established;

10) After the connection is established, AP 3 sends a "CONFIRM" message to AP 1.

\section{ILlustrative NumERICAL RESUlts}

In this section, we present the performance evaluation using NS2 (ns-2.26) with the CMU wireless extension [6]. We use $11 \mathrm{Mbps}$ channel bitrate to simulate IEEE 802.11b WLAN environment. Packet size is 512 bytes unless otherwise specified. For the radio propagation model, a two-ray path loss model is used. We do not consider fading and mobility in our simulations. We assume the transmission range of all nodes including the access point is $250 \mathrm{~m}$. All simulation results are the average

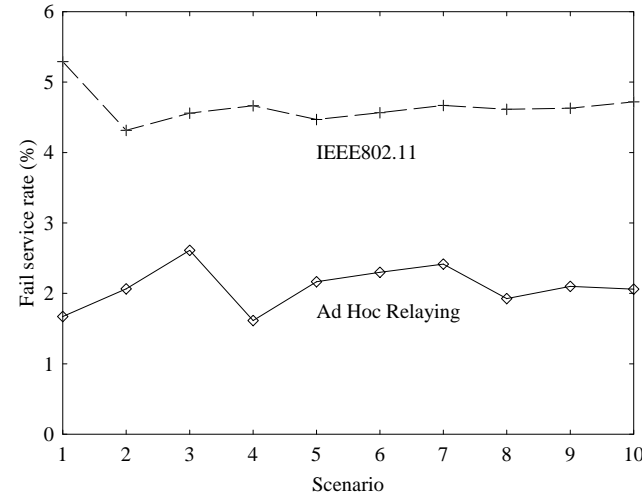

a. The lightly congested network .

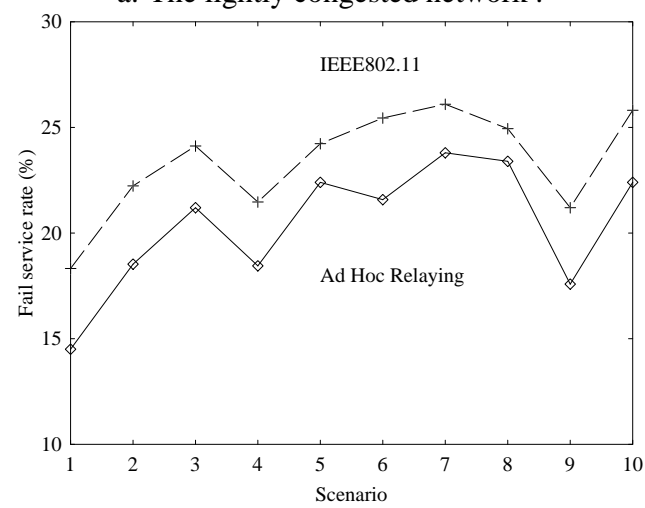

b. The heavily congested network.

Fig. 4. The failure rate of proving service to the users.

of 10 runs and each simulation runs for 10 minutes of simulation time. We use the pure WLAN performance as the baseline to compare with.

For network topology, we generate a network with three APs, the locations of which are as shown in Fig. 1 (The distances among are $250 \sqrt{2} \mathrm{~m}$.), and a number of users of random location within the transmission range of each AP. Such users arrive at each AP according to the Poisson distribution with different mean inter-arrival time $\tau_{i}$ seconds, which is smaller in the hotspot AP such that the users arrive more frequently. The holding time $t$ of the users is uniformly distributed with a mean of $H$. We use UDP traffic from the users to a AP with bandwidth requirement $B \mathrm{Kbps}$. Two different traffic conditions are generated to simulate a lightly congested network and a heavy loaded one. In this paper, we compare the performance of WLAN with and without ad hoc relaying in terms of service failure rate and channel utilization in each AP. The service failure rate is defined as the percentage of users whose requirement can not be met. The channel utilization is measured at each AP individually.

In the lightly congested network, the mean user inter-arrival times in seconds for the three APs are $\tau_{1}=20, \tau_{2}=30$ and $\tau_{3}=60$, respectively. The average holding time $H=10$ minutes and the bandwidth requirements of the users are set to $B=200 \mathrm{Kbps}$ such that the hot-spot (AP 1 ) is lightly congested while the other two are under utilized. In order to make a heavily congested network, we adjust the system parameters to be $\tau_{1}=15, \tau_{2}=20$ and $\tau_{3}=60$, respectively, such that AP 1 an AP 2 are congested while AP 3 is under utilized. Other parameters remain the same as above.

We show in Fig. 4 the service failure rate in the hot-spot network with and without ad hoc relaying connections. From the 


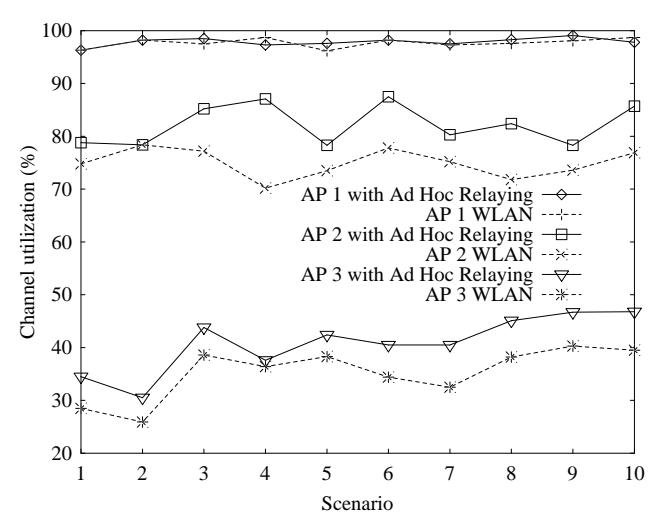

a. The lightly congested network .

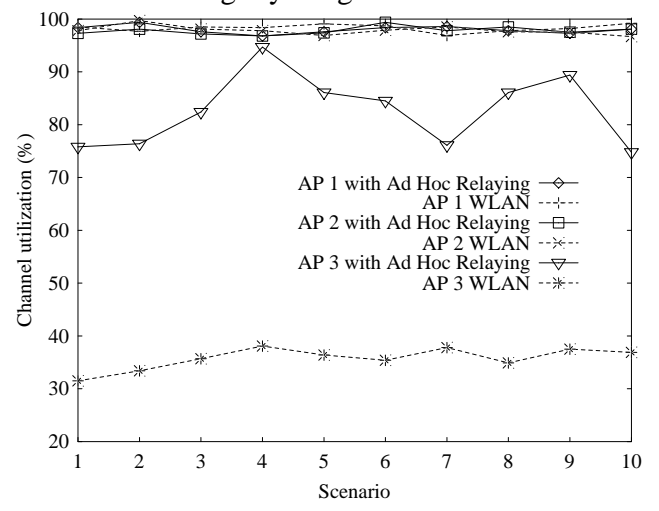

b. The heavily congested network.

Fig. 5. The channel utilization in each AP of the networks..

figures, we see that for both lightly and heavily congested networks, the ad hoc relaying scheme achieves substantially lower failure rate. In the lightly congested network (Fig. 4 a.), since the traffic load is not very heavy, only around five percent of the users cannot get their service in the hot-spot AP. With ad hoc relaying connections, most users redirected by AP 1 may get their service from AP 2 or AP 3 . Hence the failure rate is reduced by the ad hoc relaying scheme. However, some of them may not be able to establish ad hoc connections to the neighboring AP because they are too far away from the neighbor. Therefore, even with ad hoc relaying scheme, there are still some users which cannot get their service. In the heavy congested network (Fig. 4 b.), since the traffic load is heavier, both AP 1 and AP 2 are congested. More users, which cannot get their service from the congested APs, need to be redirected to the only AP (AP 3 ) that is under utilized. The users are less likely to establish connections to it. Hence the service failure rate in the heavily congested network does not decrease as much as in the lightly congested network.

In Fig. 5 we compare the channel utilization in each AP. As expected, the congested APs are fully utilized in both cases. For the other APs, which are under utilized by their own users, the channel utilization is increased with the ad hoc relaying users. Because there are only a few users need to be redirected in the light congested network, the channel utilization is increased by only a little (as shown in Fig. 5 a.). As traffic becomes heavier, the number of users being redirected increases. The channel bandwidth of all APs is highly utilized even in the AP which is under utilized without ad hoc relaying. Therefore, with ad hoc relaying scheme, the channel bandwidth is used more efficiently and the total system throughput is hence improved.

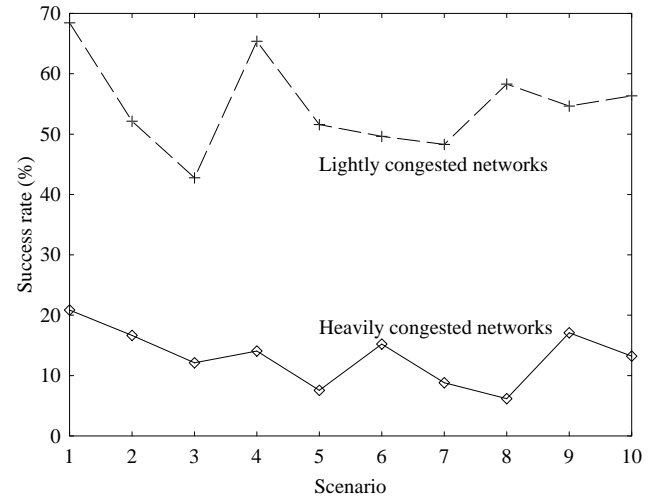

Fig. 6. The success rate of ad hoc connection establishment.

Figure 6 shows the success rate of ad hoc connection establishment in both light and heavy traffic networks. As we can see, around half of the users being redirected can connect to their neighboring APs in the light traffic network. The failures, as mentioned before, are mainly due to the coverage limitation of the agents in the neighboring APs. In the heavy congested network, the success rate is even lower. This is because there are more users being redirected and a small fraction of them can nearly use up the channel bandwidth of the neighboring AP.

In order to further increase the success rate, we are developing a new scheme which can select a proper user (maybe an already admitted one) to redirect instead of just redirecting the new user. In this way, the location (or distance) effect can be eliminated and the selected user is more likely to connect to the neighboring AP.

\section{CONCLUSION}

Due to the dynamic and unevenly distributed user load among the APs in public areas, some "hot-spots" may be congested with many users and cannot admit more requests, while their neighboring ones are serving few users and their bandwidths are under-utilized. In this paper, we propose an ad hoc relaying architecture to serve a mobile user in a hot spot by a neighboring AP, thus alleviating the congestion at the hot-spot. Simulation results show that using the scheme the failure rate is significantly reduced as well as the bandwidth utilization is improved in such hot-spot networks. In our future work, we will further investigate different schemes for implementing ad hoc relaying wireless LANs.

\section{REFERENCES}

[1] IEEE Standards Board, "Wireless LAN Medium Access Control (MAC) and Physical Layer (PHY) Specifications.” IEEE Std 802.11-1997, Nov 1997.

[2] J. Chen, S.-H. G. Chan, and J. He, "Mixed-Mode WLAN: The integration of ad hoc mode with wireless LAN infrastructure." to appear in IEEE Globecom 2003.

[3] A. Balachandran, P. Bahl, and G. M. Voelker, "Hot-spot congestion relief in public-area wireless networks," in Proc. IEEE Workshop on Mobile Computing Systems and Applications, pp. 70-80, 2002.

[4] H. Wu, C. Qiao, S. De, and O. Tonguz, "Integrated cellular and ad hoc relaying systems: iCAR," IEEE Journal on Selected Areas in Communications, vol. 19, no. 10, pp. 2105-2115, 2001.

[5] E. Y. amd O Tonguz, S. Mishra, H. Wu, and C. Qiao, "Efficient dynamic load balancing algorithms using iCAR systems: a generalized framework," in Proceedings of Vehicular Technology Conference, VTC2002, pp. 586590, IEEE, Fall 2002.

[6] "http://www.isi.edu/nsnam/ns." 